\title{
ANALYSIS OF GRAIN BOUNDARY CAVITATION DAMAGE IN COPPER
}

\author{
DAVID P. FIELD, BRENT L. ADAMS AND JUNWU ZHAO
}

Dept. of Mechanical Engineering, Yale University, New Haven, CT 06520-2157

\begin{abstract}
The interface damage function (IDF) defining the fraction of damage on crystallite interfaces in a polycrystalline material ${ }^{1}$ is extended to include functional dependence upon individual crystallite lattice orientation of grains composing a boundary. The material studied was OFHC copper crept at $10 \mathrm{MPa}$ and $0.6 \mathrm{~T}_{\mathrm{m}}$. Major peaks in the IDF were found through a series of projections of the eight-dimensional domain of the function. A maximum value of 0.95 was found for the IDF.
\end{abstract}

\section{INTRODUCTION}

Voids that form on grain boundaries of polycrystalline materials subjected to stress at elevated temperatures are generally distributed heterogeneously $1-5$. Interfaces of similar orientation to a given stress state contain differing fractions of cavitation damage. Stress state and strain rate play major roles in determining the orientation of grain boundaries which are preferentially damaged during creep ${ }^{4-7}$. However, the heterogeneity of void formation prompts further investigation into the structure of boundaries on which damage occurs. A few recent studies $1,8,9$ have suggested that crystallite lattice orientation, or more specifically lattice misorientation, is an important consideration in determining whether an interface is preferentially damaged. Adams et al $^{1}$ proposed an interface damage function (IDF) with functional dependence upon the interface normal and upon the lattice misorientation. It was suggested that damage in OFHC copper was prevalent on certain special boundaries of high group multiplicity, and that random high angle boundaries were relatively damage free. This contradicts other studies on fcc materials 8,9 which have concluded that random high angle boundaries are preferentially damaged and special CSL boundaries resist cavitation. Both conclusions may be correct for the given experimental conditions. Void nucleation and growth, however, may be a function not only of lattice misorientation, but also of each crystallite orientation in the surrounding material.

This paper focuses on creep damage as a function of the interface normal and the crystal lattice orientation of each crystallite forming the boundary. In an attempt to isolate the types of grain boundaries which are preferentially damaged, an IDF

$S_{S}^{D}\left(g, n, g^{\prime}\right)$ is proposed. $S_{S}$ is the surface area per unit surface, and the superscript " $D$ " denotes damaged. This function has a value of 0 if there is no damage on the boundaries with interface normal $\mathbf{n}$ comprised by crystallites with orientations $\mathbf{g}$ and $\mathbf{g}^{\circ}$, and has a value of 1 if the boundaries are totally separated.

\section{DETERMINATION OF AN INTERFACE DAMAGE FUNCTION}

The IDF is connected to the function $\operatorname{Sv}(g, n, g)$ (surface area of interface per unit volume) which is similar to the Interface Structure Distribution Function (ISDF) given by Adams ${ }^{10,11}$. If a function which gives the damaged surface area per unit 
volume, $S_{\mathrm{V}}^{\mathrm{D}}\left(\mathrm{g}, \mathrm{n}, \mathrm{g}^{\prime}\right)$, is considered, the IDF may be given as the quotient of two functions:

$$
S_{\mathrm{S}}^{\mathrm{D}}\left(\mathrm{g}, \mathrm{n}, \mathrm{g}^{\prime}\right)=\frac{S_{\mathrm{V}}^{\mathrm{D}}\left(\mathrm{g}, \mathrm{n}, \mathrm{g}^{\prime}\right)}{S_{\mathrm{V}}\left(\mathrm{g}, \mathrm{n}, \mathrm{g}^{\prime}\right)} .
$$

The function $S_{v}\left(g, n, g^{\prime}\right)$ may be found from a number of stereological procedures ${ }^{10-12}$. One method is to scan the microstructure with random test lines and keep track of grain boundary intersections of a given type per unit length, $P_{L}(g, g \mid t)$, where $t$ is the direction of the scan line. The fundamental equation which connects the measured function $P_{L}(g, g \mid t)$ with the desired function $\operatorname{Sv}\left(g, n, g^{\prime}\right)$ is

$$
P_{L}(g, g \mid t)=\frac{1}{2} \int S v(g, n, g)|t \cdot n| d n \text {. }
$$

This equation can be solved for $S v\left(g, n, g^{\prime}\right)$ provided sufficient $P_{L}(g, g \mid t)$ measurements are available. The function $P_{L}^{D}(g, g \mid t)$, which is the damaged intersections of $(g, g)$ type grain boundary per unit length along a scan line of direction $t$, is measured by including only those interfaces upon which a void is intersected. Using this measure and an equation similar to equation (2), we find the function $S_{v}^{D}\left(g, n, g^{\prime}\right)$.

Each of the functions in equation (2) can be represented by a series of orthogonal basis functions. The expression $|\mathbf{t} \cdot \mathbf{n}|$ is simply $|\cos \alpha|$ where $\alpha$ is the angle between $n$ and $t$, and can be expanded in a series of Legendre's polynomials. To simplify the equations we neglect dependence upon $g$ and $g^{\prime}$ and concentrate on $P_{L}(t)$ and $S_{V}(n)$. We proceed as follows (compare with Hilliard ${ }^{12}$ ):

$$
\begin{aligned}
& S V(n)=\sum_{r=0}^{\infty} \sum_{t=-2 r}^{2 r} S_{2 r}^{t} K_{2 r}^{t}(n) \\
& P_{L}(t)=\sum_{r=0}^{\infty} \sum_{t=-2 r}^{2 r} S_{2 r}^{t} K_{2 r}^{t}(t)
\end{aligned}
$$

where $K_{2 r}^{t}(n)$ are the surface spherical harmonic functions and $S_{2 r}^{t}$ and $S_{2 r}^{\prime t}$ are constant coefficients defining the functions. Combining equations (2) and (3) and representing $|\cos \alpha|$ in a series expansion with coefficients $A_{2} r^{\prime}$ we get

$$
P_{L}(t)=\frac{1}{2} \int \sum_{r=0}^{\infty} \sum_{t=-2 r}^{2 r} S_{2 r}^{t} K_{2 r}^{t}(n) \sum_{r^{\prime}=0}^{\infty} A_{2 r^{\prime}} P_{2 r^{r}}(\cos \alpha) d n
$$

where $\mathrm{P}_{2 \mathrm{r}}(\cos \alpha)$ are Legendre's polynomials. The solution to equation (5) is found by defining a new function

$$
V_{2 r}(n)=\sum_{t=-2 r}^{2 r} S_{2 r}^{t} K_{2 r}^{t}(n)
$$

and substituting $V_{2 r}(n)$ back into equation (5) to obtain ${ }^{13}$ 


$$
P_{L}(t)=\frac{1}{2} \int \sum_{r=0}^{\infty} V_{2 r}(n) \sum_{r^{\prime}=0}^{\infty} A_{2 r^{\prime}} P_{2 r^{\prime}}(\cos \alpha) d n=\sum_{r=0}^{\infty} \sum_{r^{\prime}=0}^{\infty} \frac{2 \pi}{4 r+1} A_{2 r^{\prime}} V_{2 r}(t) \delta_{r r^{\prime}}
$$

where $\delta_{r^{\prime}}$ is the Kronecker delta. From equations (4) and (7) we have

which gives the result

$$
P_{L}(t)=\sum_{r=0}^{\infty} \sum_{t=-2 r}^{2 r} S_{2 r}^{\prime t} K_{2 r}^{t}(t)=\sum_{r=0}^{\infty} \frac{2 \pi}{4 r+1} A_{2 r} \sum_{t=-2 r}^{2 r} S_{2 r}^{t} K_{2 r}^{t}(t)
$$

$$
S_{2 r}^{t}=S_{2 r}^{t} \frac{4 r+1}{2 \pi} \frac{1}{A_{2 r}}
$$

The $S_{2 r}^{t}$ coefficients are found from the measured function $P_{L}(t)$ and the coefficients defining $S_{v}(n)$ are given by expression (9). If the functional dependence on $g$ and $g$ is now included, equations (3) and (4) become

$$
\begin{aligned}
& \operatorname{Sv}\left(\mathrm{g}, \mathrm{n}, \mathrm{g}^{\prime}\right)=\sum_{\mathrm{r}=0}^{\infty} \sum_{\mathrm{t}=-2 \mathrm{r}}^{2 \mathrm{r}} \sum_{l=0}^{\infty} \sum_{\mu=1}^{\mathrm{M}(l)} \sum_{\mathrm{n}=-l}^{l} \sum_{l^{\prime}=0}^{\infty} \sum_{\mu^{\prime}=1}^{\mathrm{M}(l)} \sum_{\mathrm{n}^{\prime}=-l}^{l}{ }_{l \mu \mathrm{n}}^{l_{\mu^{\prime} \mathrm{n}^{\prime}}} \mathrm{s}_{2 \mathrm{r}}^{\mathrm{t}} \mathrm{K}_{2 \mathrm{r}}^{\mathrm{t}}(\mathrm{n}) \stackrel{\mathrm{T}}{\mathrm{T}}_{l}^{\mu \mathrm{n}}(\mathrm{g}) \dot{\mathrm{T}}_{l^{\prime}}^{\mu^{\prime} \mathrm{n}^{\prime}}\left(\mathrm{g}^{\prime}\right)
\end{aligned}
$$

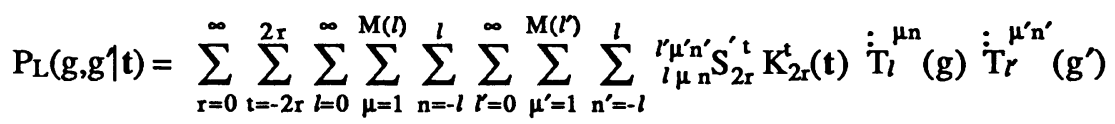

respectively. The $\dot{\mathrm{T}}_{l}^{\mu \mathrm{n}}(\mathrm{g})$ functions are the symmetric generalized spherical harmonics as given by Bunge ${ }^{14}$. The relationship between the coefficients defining the functions $P_{L}(g, g \mid t)$ and $\operatorname{Sv}\left(g, n, g^{\prime}\right)$ remains unchanged

$$
{ }_{l \mu \mathrm{n}}^{l \mu^{\prime} \mathrm{n}^{\prime}} S_{2 \mathrm{r}}^{t}={ }_{l \mu \mathrm{n}}^{l_{\mu^{\prime} \mathrm{n}^{\prime}}^{\prime}} S_{2 \mathrm{r}}^{t} \frac{4 \mathrm{r}+1}{2 \pi} \frac{1}{\mathrm{~A}_{2 \mathrm{r}}}
$$

\section{EXPERIMENTAL DETAILS}

Half-hard OFHC alloy 101 copper plate (99.99\% pure) with a thickness of $1.27 \mathrm{~cm}$ was machined into cylindrical tensile specimens with a gage section diameter of 6.35 $\mathrm{mm}$. The axis of each specimen was aligned with the rolling direction in the plate. The specimens were annealed at $900^{\circ} \mathrm{C}$ in a vacuum of $10^{-6}$ torr for two hours and furnace cooled. The resulting grain size was $165 \mu \mathrm{m}$. Creep experiments in uniaxial-axial tension with a stress of $10 \mathrm{MPa}$ were performed using a dead-weight loading apparatus. The specimens were crept at $540^{\circ} \mathrm{C}\left(0.6 \mathrm{~T}_{\mathrm{m}}\right)$ for 48 hours $\left(\approx 0.6 \mathrm{t}_{\mathrm{r}}\right)$. The axial strain from the creep tests was less than 2 percent.

To analyze and quantify the grain boundary damage, line scans were made through the gage sections of the crept specimens and the functions $P_{L}(g, g \mid t)$ and $P_{L}^{D}(g, g \mid t)$ were measured. This was enabled by cutting several plane sections through the specimens. The cuts were made using a spark cutter and care was taken to minimize damage to the microstructure as the cuts were made. The depth of damage on the plane surfaces from use of the spark cutter is estimated at $1 \mu \mathrm{m}$. The cutting was followed by a slight electrochemical polish which removed all surface damage caused by the spark cutter and yet care was taken so as to not significantly effect the cavities on the plane section.

To measure the functions $P_{L}(g, g \mid t)$ and $P_{L}^{D}(g, g \mid t)$, each plane section described above was divided into small regions. From each region a grain map was constructed by 
manually digitizing all grain boundaries. The boundaries were defined by line segments which separated crystallites of differing orientations. The damage on a boundary was defined by yet another set of line segments. Voids on the interfaces were defined as damage if they exceeded $1 \mu \mathrm{m}$ in diameter. Figure 1 shows a representative section of a digitized grain map. The bold lines denote damage to that portion of the boundary. The orientation of each crystallite was measured using the Back-scattered Kikuchi Diffraction (BKD) pattern method 15,16 and a point within each grain corresponding to a given orientation was manually digitized. Line scans were made in several directions across each section with a perpendicular distance of $100 \mu \mathrm{m}$ between parallel scan lines. Data files were generated which contained the scan direction and the crystallite orientation in Euler's angles for each grain on each boundary intersected. There were 2,100 individual lattice orientations with about 10,000 grain boundaries digitized. This resulted in approximately 40,000 grain boundary intersections

The computational requirements involved in determining the function $\operatorname{Sv}\left(g, n, g^{\prime}\right)$ are extensive in that the coefficients of the series expansion must first be found from several thousand data points. Finding the value of the function after the coefficients are calculated is computationally difficult because the summation in equation (10) must be performed for each set of variables in the function. To enable the calculation of the expansion coefficients a maximum value of four was chosen for $r$ and a maximum value of eight for $l$ and $l$. The value of $\mathrm{M}(l)$ for cubic symmetry when $l$ is less than 12 is always one.

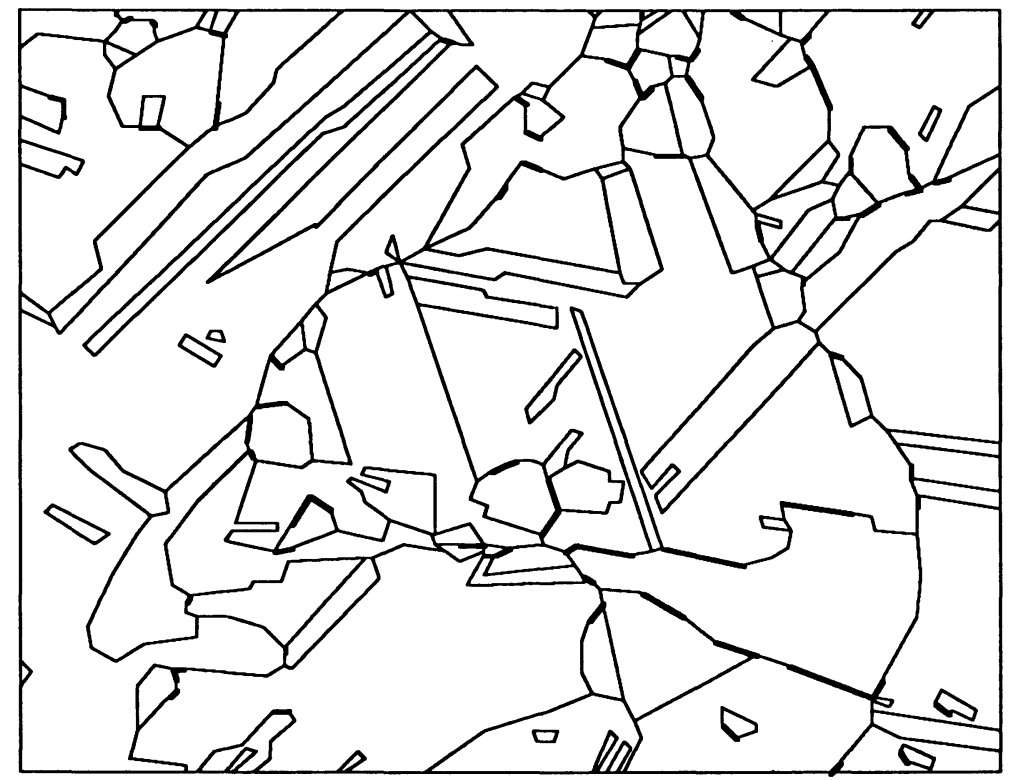

Figure 1 - Representative grain map. The bold lines indicate damaged boundaries. 


\section{DISCUSSION OF RESULTS}

We wish to discover the structure of interfaces which are the most readily damaged in the creep experiments. Several sections were made through the domain of the IDF and examined for critical values of the independent variables. These variables were held constant and the value of the IDF was found at several discrete points within the reduced domain of the function. By integrating the IDF over the spaces of $\mathbf{g}$ and $\mathbf{g}^{\mathbf{b}}$, we determined the grain boundary normal orientation of the interfaces which were

preferentially damaged, $S_{S}^{D}(n)$. The maximum value of this function was found to be nearly aligned with the tensile (rolling) direction of the specimens. Setting the interface to be normal to the tensile axis reduces the space of the IDF to two sets of Euler angles. Considering only the fundamental region of Euler space for cubic crystal symmetry, the space of the function remains unmanageably large.

Consider the orientation of a single crystallite which has a damaged boundary.

Integrating the IDF over the space of $g^{\prime}$ creates the function $S_{S}^{D}(g, n)$. This function is the fraction of damaged interfaces which have an adjoining crystallite with orientation g. The maximum value of this function was 0.9403 and occured at an orientation of $\left(\varphi_{1}=140^{\circ}, \Phi=70^{\circ}, \varphi_{2}=70^{\circ}\right)$. However, by limiting the calculation of the function

$S_{S}^{D}(g, n)$ to only those areas of $S_{V}(g, n)$ which are greater than 0.0001 , the major peaks of this new function were lost and the maximum value became 0.4708 at $(310,50,10)$. The latter calculation is more reliable because it suggests that when few boundaries of a given type are present in the structure, we cannot find the statistical fraction which are damaged. This is especially true with the limited data available.

Using the above results we can calculate a portion of the complete IDF from the experimental data by holding the interface normal constant and fixing the orientation of one crystallite at $(310,50,10)$. We again limit the calculation of the IDF to the areas of $S_{\mathrm{V}}(310,50,10,0,0, \mathrm{~g})$ which are greater than ten percent of the maximum peak value in that function. In this portion of the IDF we get a maximum value of .9490 at a $g^{\prime}$ 'orientation of $(190,70,60)$ (see Figure 2$)$. The misorientation between these two crystallites is approximately $(33,84,31)$. This is a special boundary of group multiplicity 4 as identified by Zhao and Adams ${ }^{17}$.

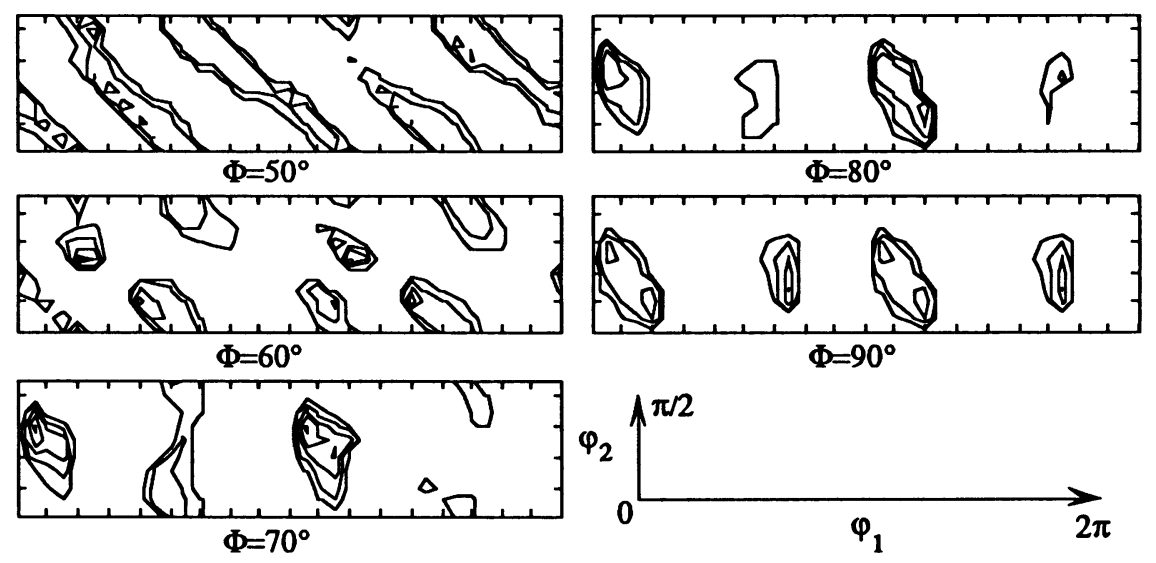

Figure 2 - A section of the measured IDF $S_{S}^{D}\left(310,50,10,0,0, \varphi_{1}, \Phi, \varphi_{2}\right)$. 


\section{CONCLUSIONS}

There are two primary challenges in this type of analysis of damaged interfaces. First, statistical reliability requires an enormous data set. Second, locating peaks in the IDF and determining whether or not they are significant is difficult because of the large domain of the function.

In searching through the space of the IDF we have discovered several types of grain boundaries which are preferentially damaged. For $10 \mathrm{MPa}$ stress and at a temperature of .6 $\mathrm{Tm}$, OFHC copper sheet tends to damage on grain boundaries which are normal to the tensile axis. Crystallites with lattice orientation of $\left(\varphi_{1}=310^{\circ}, \Phi=50^{\circ}, \varphi_{2}=10^{\circ}\right)$ have a significant fraction, 47 percent, of damaged grain boundaries regardless of the neighboring crystallite when the boundary is normal to the tensile axis. The highest fraction of damage observed was 0.95 for boundaries with structure $\left(\mathrm{g}, \mathrm{n}, \mathrm{g}^{\prime}\right)=$ $(310,50,10,0,0,190,70,60)$.

The method used to obtain the stereological data necessary in this analysis was extremely time consuming and collecting several million data points is not feasable. With anticipated innovations in the procedure to collect the data (ie: automated indexing of BKD patterns and computer driven line scanning) the IDF could be a valuable tool in finding which boundaries are preferentially damaged. From the calculation of an IDF one has the information necessary to more completely consider the dominant mechanisms responsible for void nucleation and growth. The IDF also gives detailed information about of interfaces which should be avoided in a microstructure subjected to given temperature and loading conditions.

\section{ACKNOWLEDGEMENTS}

This research was funded by the Office of Basic Energy Sciences of the United States Department of Energy (DE-FG02-88ER45355).

\section{REFERENCES}

1. B. L. Adams, J. Zhao and D. O'Hara, Acta metall. mater. 38, 953 (1990).

2. B. F. Dyson, Metal Sci. 10, 349 (1976).

3. J. R. Rice, Acta Met 29, 675 (1981).

4. R. W. Evans and B. Wilshire, Creep of Metals and Alloys, Pineridge Press, Swansea (1985).

5. A. C. F. Cocks and M. F. Ashby, Prog in Mat. Sci. 27, 189 (1982).

6. A. S. Argon, Recent Advances in Creep and Fracture of Engineering Materials and Structures, Pineridge Press, Swansea (1982).

7. B. F. Dyson, Scripta Met. 17, 31 (1983).

8. T. Watanabe, Metall. Trans. 14A, 531 (1983).

9. J. Don and S. Majumdar, Acta Met 34, 961 (1986).

10. B. L. Adams, Metall. Trans. 17A, 2199 (1986).

11. J. Zhao, J. S. Koontz and B. L. Adams, Metall. Trans. 19A, 1179 (1988).

12. J. E. Hilliard, Trans. Am. Inst. Min. Engrs. 224, 1201 (1963).

13. E.W. Hobson, The Theory of Spherical and Ellipsoidal Harmonics, Chelsea Publishing Company, New York, 1955.

14. Bunge, H.J., Mathematische Methoden der Texturanalyse, Akademie-Verlag, Berlin, 1969.

15. J. A. Venables and C. J. Harland, Phil Mag 27, 1193 (1973).

16. D. J. Dingley and K. Bab-Kishi, Scanning Electron Microscopy II, 383 (1986).

17. J. Zhao and B. L. Adams, Acta crystallogr. A44, 326 (1988). 